OPEN ACCESS

Edited by:

Zhiyong Li,

Shanghai Jiao Tong University, China

Reviewed by:

Anusorn Cherdthong

Khon Kaen University, Thailand

Xueping Han,

Northwest Institute of Plateau Biology,

Chinese Academy of Sciences (CAS),

China

*Correspondence:

Chengfu Zhang

tibetzcf@126.cn

Kai Cui

cuikai@caas.cn

${ }^{\dagger}$ These authors have contributed equally to this work

Specialty section: This article was submitted to

Microbial Symbioses,

a section of the journa

Frontiers in Microbiology

Received: 02 November 2021 Accepted: 20 December 2021

Published: 10 February 2022

Citation:

Han L, Xue W, Cao H, Chen X, Qi F, Ma T, Tu Y, Diao Q, Zhang C and Cui K (2022) Comparison of Rumen Fermentation Parameters and Microbiota of Yaks From Different

Altitude Regions in Tibet, China.

Front. Microbiol. 12:807512.

doi: 10.3389/fmicb.2021.807512

\section{Comparison of Rumen Fermentation Parameters and Microbiota of Yaks From Different Altitude Regions in Tibet, China}

\author{
Lulu Han ${ }^{1+}$, Wanchao Xue ${ }^{2+}$, Hanwen $\mathrm{Cao}^{3}$, Xiaoying Chen ${ }^{3}$, Fasheng Qi ${ }^{4}$, Tao $\mathrm{Ma}^{1}$, \\ Yan Tu', Qiyu Diao', Chengfu Zhang ${ }^{3 *}$ and Kai Cui ${ }^{1,3 *}$
}

\footnotetext{
${ }^{1}$ Key Laboratory of Feed Biotechnology of the Ministry of Agriculture and Rural Affairs, Institute of Feed Research of the Chinese Academy of Agricultural Sciences, Beijing, China, ${ }^{2}$ Animal Husbandry and Veterinary Station of Huangyuan, Huangyuan, China, ${ }^{3}$ State Key Laboratory of Hulless Barley and Yak Germplasm Resources and Genetic Improvement, Institute of Animal Science and Veterinary, Tibet Academy of Agricultural and Animal Husbandry Sciences, Lhasa, China, ${ }^{4}$ General Station of Animal Husbandry and Veterinary Technology Extension of Naqu, Naqu, China
}

Rumen microbiota are closely linked to feed utilization and environmental adaptability of ruminants. At present, little is known about the influence of different extreme environments on the rumen microbiota of yaks. In this study, 30 ruminal fluid samples from 30 healthy female yaks (average $280 \mathrm{~kg}$ of BW) in 5-8 years of life were collected from three regions in Tibet, China, and compared by gas chromatography and highthroughput sequencing. Results showed that propionic acid, butyric acid, and total volatile fatty acids were significantly $(p<0.05)$ higher, while microbial abundance and diversity were significantly $(p<0.05)$ lower, in the Nagqu $(4,500 \mathrm{~m}$ altitude) compared with the Xigatse $(4,800 \mathrm{~m}$ altitude) and Lhasa $(3,800 \mathrm{~m}$ altitude) regions. Principal coordinate analysis revealed significant $(p<0.05)$ differences in rumen microbial composition of yaks from different regions. Specifically, Bacteroidetes and Firmicutes were identified by linear discriminant analysis effect size (LDA > 3) as being the signature phyla for Xigatse and Nagqu regions, respectively. In addition, the relative abundance of Rikenellaceae_RC9_gut_group, Quinella, Prevotellaceae_UCG003, Lachnospiraceae_NK3A20_group, Papillibacter, Ruminococcaceae_UCG-010, Prevotellaceae_NK3B31_group, and Ruminococcaceae_UCG-005 correlated with altitude and rumen fermentation parameters $(p<0.05)$. Finally, the predicted function of rumen microbiota was found to differ between regions $(p<0.05)$. In summary, our results reveal that regions located at different altitudes influence microbiota composition and fermentation function of yaks' rumen. The present findings can provide mechanistic insights on yak adaptation to high altitudes and improve the feeding efficiency of these animals in extreme regions.

Keywords: yak, altitude, region, rumen, microbiota 


\section{INTRODUCTION}

Yaks (Bos grunniens) are long-haired ruminants living in highaltitude regions $(>3,000 \mathrm{~m}$ ) such as the Tibetan Plateau in China (Guo et al., 2015). About $90 \%$ of the world population of yaks is found in China (Li et al., 2018), where these iconic animals provide meat, milk, wool, and fuel for the local nomadic people (Liu et al., 2008; Long et al., 2008; Qiu et al., 2012; Gao et al., 2013; Zhang J. et al., 2020), who call yaks "boats of the plateau" (Liu et al., 2019).

Owing to the extreme environmental conditions of the Tibetan Plateau and long-term natural selection, yaks have developed a tolerance for cold, low oxygen, rough feeding, and hardship (Long et al., 2008; Qiu et al., 2012). Different environments lead to different metabolic strategies, which allow animals to adapt and minimize the adverse effects of environmental changes (Whiteley and Faulkner, 2005; Oliveira et al., 2021). Recent evidence suggests that such effect is largely due to the microbiota in the digestive tract, which play an important role in nutrient intake, metabolism, and immune response of the host (Furman et al., 2020; O'Hara et al., 2020; Bi et al., 2021). Consequently, the correlation between the adaptability of yaks to environmental changes and their gastrointestinal microbiota composition has attracted increasing attention. Guo et al. (2021) found that the fecal microbiota of yaks reflected seasonal dietary changes, allowing yaks to make better use of low-protein roughage. Whereas fecal microbiota composition of yaks differs across regions, its functional profile is more uniform (Liu et al., 2021). The rumen is one of the most important digestive organs of ruminants and is characterized by an abundance of microbiota (Guan et al., 2008; Khan et al., 2016). Indeed, owing to their microbiota, ruminants can ferment solid feed into volatile fatty acids (VFAs), which provide $70 \%$ of the required energy (Anantasook et al., 2013; Khan et al., 2016). At the same time, rumen microbiota and VFAs are the main factors explaining different feed efficiencies among individual cattle (Liang et al., 2017; Zhou et al., 2018; Zhang Y. et al., 2020). Although Wu et al. (2021) found that altitude affected the composition of rumen microbiota in yaks, it remains to be determined how microbiota and VFAs vary under extreme conditions (>3,000 m altitude).

To understand the response of yak rumen microbiota and VFAs to different altitudes, the present study employed microbiome technology to characterize rumen composition in yaks living at different altitudes in three extreme regions of Tibet. We hypothesized that rumen fermentation parameters and microbiota of yaks would change dynamically with increasing altitude.

\section{MATERIALS AND METHODS}

The experimental protocol was approved by the Chinese Academy of Agricultural Sciences Animal Ethics Committee, and all procedures were performed in accordance with humane animal care and handling (AEC-CAAS-20190905).

\section{Study Regions, Animals, and Experimental Design}

Three study regions, including Zhongba County, Xigatse City $\left(84^{\circ} 03^{\prime} \mathrm{E}, 29^{\circ} 77^{\prime} \mathrm{N}\right.$; about $4,800 \mathrm{~m}$ altitude, HAL), Nagqu City $\left(92^{\circ} 07^{\prime} \mathrm{E}, 31^{\circ} 48^{\prime} \mathrm{N}\right.$; about $4,500 \mathrm{~m}$ altitude, MAL), and Dangxiong County, Lhasa City $\left(91^{\circ} 05^{\prime} \mathrm{E}, 30^{\circ} 51^{\prime} \mathrm{N}\right.$; about 3,800 $\mathrm{m}$ altitude, LAL), were selected. The three regions are key breeding grounds for yaks; their average annual air temperature is $-4.0^{\circ} \mathrm{C},-0.5^{\circ} \mathrm{C}$, and $2.0^{\circ} \mathrm{C}$, while precipitation is 280,433 , and $485 \mathrm{~mm}$, respectively.

In September 2019, ten healthy female yaks (average $280 \mathrm{~kg}$ of BW) in 5-8 years of life were randomly selected from every study region. At the study regions, yaks are allowed to live free-range, follow their dams, and are not given any artificial feed, relying instead on typical alpine meadows as the main type of vegetation.

\section{Ruminal Chyme Collection and Analysis of Fermentation Parameters}

Before morning grazing, ruminal chyme (30 samples in total) was collected through the mouth of yaks using an esophageal tube as described previously (Ramos-Morales et al., 2014). Next, the chyme was filtered through a four-layer woven gauze to collect the ruminal fluid, which was immediately frozen in liquid nitrogen $\left(-80^{\circ} \mathrm{C}\right)$ for subsequent microbiota and fermentation characterization. The $\mathrm{pH}$ value was detected using a portable pH meter (206-pH1; Testo). For VFA analysis, three tubes containing $1 \mathrm{ml}$ of rumen fluid were taken from each sample after thawing and centrifugation at $20,000 \times g$ and $4^{\circ} \mathrm{C}$ for $15 \mathrm{~min}$. Then, $0.25 \mathrm{ml}$ metaphosphoric acid $(25 \mathrm{~g} / 100 \mathrm{ml})$ was added per tube and the sample was analyzed by gas chromatography equipping a megabore HP-MOLSIV column (film thickness: $30 \mathrm{~m} \times 0.53 \mathrm{~mm} \times 25 \mu \mathrm{m})($ SP-3420A; Beifenrili Analyzer Associates, Beijing, China) (Yue et al., 2009).

\section{DNA Extraction, PCR Amplification, and 16S rRNA Sequencing}

Microbial DNA was extracted from rumen fluid samples (30 samples in total) using PowerSoil DNA Isolation Kit (MoBio Laboratories, Carlsbad, CA) following the manufacturer's guidelines. The concentration and purity of the extracted DNA were measured by a NanoDrop 2000 Spectrophotometer (Thermo Fisher Scientific, Waltham, MA, United States). The V3-V4 region of the bacterial 16S rRNA gene was amplified using primer $338 \mathrm{~F}$ ( $5^{\prime}$-ACTCCTACGGGAGGCAGCAG-3') and 806R (5'-GGACTACHVGGGTWTCTAAT-3') (Takahashi et al., 2014). PCR amplification proceeded through an initial denaturing step at $94^{\circ} \mathrm{C}$ for $5 \mathrm{~min} ; 28$ cycles at $94^{\circ} \mathrm{C}$ for $30 \mathrm{~s}$, $55^{\circ} \mathrm{C}$ for $30 \mathrm{~s}$, and $72^{\circ} \mathrm{C}$ for $60 \mathrm{~s}$; and a final extension at $72^{\circ} \mathrm{C}$ for 7 min using $25-\mu 1$ reaction volumes, containing $12.5 \mu 12 \times$ Taq PCR MasterMix, $3 \mu \mathrm{l}$ BSA $(2 \mathrm{ng} / \mu \mathrm{l}), 1 \mu \mathrm{l}$ forward primer (5 $\mu \mathrm{M}), 1 \mu \mathrm{l}$ reverse primer $(5 \mu \mathrm{M}), 2 \mu \mathrm{l}$ template DNA, and 5.5 $\mu \mathrm{lddH} 2 \mathrm{O}$. The PCR products were detected by $2 \%$ agarose gel electrophoresis, purified with the Agencourt AMPure XP kit (Beckman Coulter, La Brea, CA, United States) according to the manufacturer's instructions, and quantified by QuantiFluor-ST (Promega, Madison, WI, United States). Purified amplicons 
were sequenced on an Illumina MiSeq-PE300 platform (Illumina Inc., San Diego, CA, United States), generating $2 \times 300$ bp paired-end reads.

\section{Sequence Analysis}

The obtained paired-end reads in the original DNA fragments were merged using Flash version 1.20 (Mago and Salzberg, 2011), and then each sample was separated according to a unique barcode. After removing barcodes, primers, and splice variants, raw reads were obtained. To generate high-quality reads, a specific sliding window strategy was adopted for Trimmomatic version 0.36 , the window size was set to $50 \mathrm{bp}$, the average quality value was 20 , and the minimum reserved sequence length was $120 \mathrm{bp}$; in addition, sequences containing $\mathrm{N}$ were removed by Pear version 0.9.6 (Bolger et al., 2014). Afterward, Flash and Pear were used to merge the sequences at both ends according to PE overlap correlation, the minimum overlap was set to $10 \mathrm{bp}$, and the mismatch rate was 0.1 , allowing for fasta sequences to be obtained. Finally, the chimera containing fasta sequences was eliminated by comparison with the Gold database using the UCHIME algorithm, whereas the unknown database was removed by de novo means (Knight, 2011). Simultaneously, the short sequences, failing to meet the requirements, were removed, while high-quality sequences of clean reads were obtained. Subsequently, these clean tags were clustered into operational taxonomic units (OTUs) based on a 97\% sequence similarity threshold using the UPARSE algorithm in Vsearch version 2.7.1. In each OTU, the richest sequence was filtered as the representative sequence (Edgar, 2013). To derive species classification information corresponding to each OTU, the representative sequences were compared and analyzed by the RDP Classifier algorithm version 2.2 (Wang, 2007) and Silva database $^{1}$ (Quast et al., 2012), allowing community annotation at kingdom, phylum, class, order, family, and genus level. After the sample number of the lowest sequence was flattened, alpha diversity (Chao1, Shannon, Simpson, and observed species) was calculated by QIIME 2, while intergroup alpha index variability was demonstrated by the Kruskal-Wallis test in $\mathrm{R}$ version 4.0.2 (Miller et al., 2016). Principal coordinate analysis (PCoA) based on Bray-Curtis dissimilarity matrices and QIIME 2 was used to calculate differences in bacterial communities among groups. The linear discriminant analysis effect size (LEfSe, LDA > 3) was used to identify significant bacteria among the three groups (Miller et al., 2016). PICRUSt2 software was used to predict microbiota function and explore differences among the three groups (Douglas et al., 2019).

Rumen fermentation parameters were analyzed using oneway ANOVA and Duncan's multiple comparison in SPSS 22.0 software (IBM, Chicago, IL, United States). Differences in alpha diversity, relative abundance at phylum, family, and genus level, as well as microbiota function among the three groups were tested using the Kruskal-Wallis method in R version 4.0.3. PCoA, Venn, column, and LEfSe results were visualized using the "ape" and "ggplot2," "limma," "ggplot2," and "ggtree" packages in R, respectively. The "corrplot" package in $\mathrm{R}$ was used to analyze

${ }^{1}$ http://www.arb-silva.de the correlation between the top 20 genera of all samples, rumen fermentation parameters, and altitude (based on Spearman's coefficient). The network containing the top 20 genera was visualized using the "igraph" package in $\mathrm{R}$ version 4.0.3. All data are reported as means, and intergroup differences of $p<0.05$ were considered significant.

\section{RESULTS}

\section{Rumen Fermentation Parameters of Yaks Living in Different Regions}

As reported in Table 1, rumen fermentation parameters differed significantly between the three groups $(p<0.05)$, whereas the $\mathrm{pH}$ value had no difference between the three groups $(p>0.05)$. Also, the MAL group exhibited the highest $(p<0.05)$ total VFA content and molar proportions of propionic and butyric acids, whereas the molar proportion of acetic acid and A/P (acetic acid/propionic acid) were lower $(p<0.05)$ than in other groups. Interestingly, rumen fermentation parameters were similar between HAL and LAL groups $(p>0.05)$.

\section{Taxonomic Composition of Rumen Bacteria}

Gradual stabilization of the OTU rank curve chart indicates high coverage of test samples (Supplementary Figure 1). The rumen microbiota consisted of 5,491,884 high-quality reads, with an average of 183,063 reads per sample. A total of 7,977 OTUs were obtained based on $97 \%$ nucleotide sequence identification: 6,895 OTUs in HAL, 5,544 in MAL, and 6,109 in LAL groups (Figure 1A). Of these, 4,219 OTUs $(52.89 \%$ of the total) were shared among samples from different groups. Alpha diversity calculations (Table 2) revealed significantly higher indices of Shannon and community richness (Chaol and observed species) in HAL and LAL groups compared with the MAL group $(p<0.05)$, whereas no significant difference was detected between HAL and LAL groups $(p>0.05)$. Interestingly, we found Simpson index in HAL significantly higher $(p<0.05)$ than MAL group, whereas no significant difference was detected between LAL and other groups $(p>0.05)$. PCoA plots of bacterial structure profiles (Figure 1B) revealed distinct clustering of the three regions. The segregation and dissimilarities observed at OTU level were revealed using Bray-Curtis matrices (PERMANOVA, $p<0.001$ ).

Next, we proceeded with bacterial identification of the 30 samples at different levels. At the phylum level, the top five ruminal bacteria of yaks across all samples were Bacteroidetes (50.69\%), Firmicutes (41.11\%), Proteobacteria (1.45\%), Tenericutes (1.40\%), and Actinobacteria (1.05\%) (Figure 2A and Supplementary Table 1). The MAL group presented the lowest $(p<0.05)$ relative abundances of Bacteroidetes and Verrucomicrobia, but the highest $(p<0.05)$ relative abundance of Firmicutes compared with the HAL and LAL groups. At the family level, Prevotellaceae (24.67\%) was the most abundant taxon, followed by Ruminococcaceae (14.76\%) and Rikenellaceae (11.67\%) across all samples; other 
TABLE 1 | Effect of regions located at distinct altitudes on yak rumen fermentation parameters.

\begin{tabular}{|c|c|c|c|c|c|}
\hline \multirow[t]{2}{*}{ Parameters } & \multicolumn{3}{|c|}{ Groups $^{1}$} & \multirow[t]{2}{*}{ SEM } & \multirow[t]{2}{*}{$P$-value } \\
\hline & HAL & MAL & LAL & & \\
\hline $\mathrm{PH}$ & 6.41 & 6.54 & 6.45 & 0.042 & 0.834 \\
\hline T-VFA (mmol/L) & $49.62^{b}$ & $69.41^{a}$ & $45.22^{b}$ & 2.943 & $<0.001$ \\
\hline Acetic acid (\%) & $74.58^{a}$ & $61.73^{b}$ & $72.09^{a}$ & 0.011 & $<0.001$ \\
\hline Propionic acid (\%) & $15.88^{b}$ & $24.46^{a}$ & $18.21^{b}$ & 0.008 & $<0.001$ \\
\hline Butyric acid (\%) & $9.54^{b}$ & $13.81^{a}$ & $9.70^{b}$ & 0.005 & $<0.001$ \\
\hline $\mathrm{A} / \mathrm{P}$ & $4.73^{a}$ & $2.57^{b}$ & $3.97^{a}$ & 0.18 & $<0.001$ \\
\hline
\end{tabular}

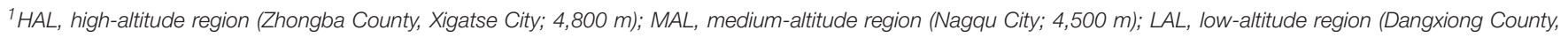
Lhasa City; 3,800 m). T-VFA, total volatile fatty acids; A/P, acetic acid/propionic acid.

Different superscript letters in the same row denote significant differences $(p<0.05)$.
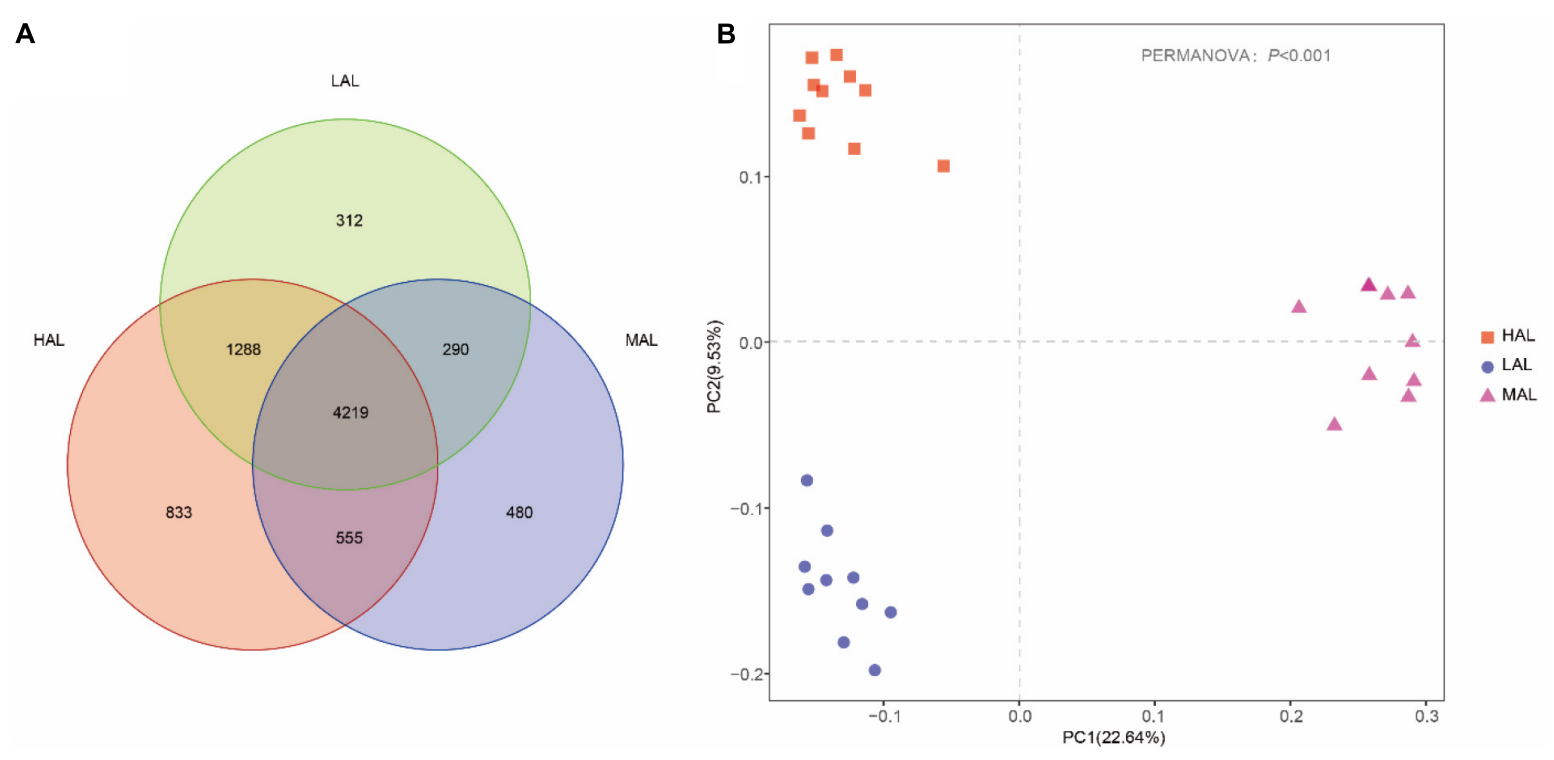

FIGURE 1 | Response of rumen microbiota from yaks among the three regions. (A) Composition of rumen microbiota from yaks inhabiting regions located at distinct altitudes (OTU-level analysis). (B) PCoA analysis of rumen microbiota from yaks inhabiting regions located at distinct altitudes. HAL, high-altitude region (Zhongba County, Xigatse City; 4,800 m); MAL, medium-altitude region (Nagqu City; 4,500 m); LAL, low-altitude region (Dangxiong County, Lhasa City; 3,800 m).

TABLE 2 | Effect of regions located at distinct altitudes on alpha diversity of yak rumen microbiota.

\begin{tabular}{|c|c|c|c|c|c|}
\hline \multirow[t]{2}{*}{ Indices } & \multicolumn{3}{|c|}{ Groups $^{1}$} & \multirow[t]{2}{*}{ SEM } & \multirow[t]{2}{*}{$P$-value } \\
\hline & HAL & MAL & LAL & & \\
\hline Chao1 & $4373^{a}$ & $3431.5^{b}$ & $4203.7^{a}$ & 168.51 & $<0.001$ \\
\hline Observed_species & $3408.6^{a}$ & $2621.1^{b}$ & $3234.6^{a}$ & 157.28 & $<0.001$ \\
\hline Shannon & $9.28^{a}$ & $8.53^{b}$ & $9.19^{a}$ & 0.27 & $<0.001$ \\
\hline Simpson & $0.994^{a}$ & $0.987^{b}$ & $0.991^{a b}$ & 0.003 & $<0.001$ \\
\hline
\end{tabular}

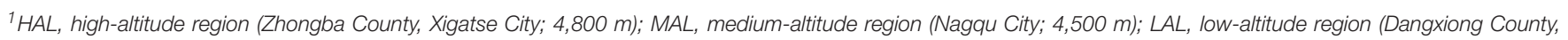
Lhasa City; 3,800 m).

Different superscript letters in the same row denote significant differences $(p<0.05)$.

families included Lachnospiraceae (9.15\%), Christensenellaceae (8.42\%), Bacteroidales_BS11_gut_group (7.20\%), Veillonellaceae (4.05\%), and Bacteroidales_S24-7_group (3.44\%) (Figure 2B and Supplementary Table 2 ). Notably, the relative abundances of Lachnospiraceae, Christensenellaceae, and Veillonellaceae were higher $(p<0.05)$ in the MAL compared with other groups, whereas the relative abundance of Rikenellaceae increased significantly $(p<0.05)$ according to the following order: MAL $<$ LAL $<$ HAL. At the genus level, the dominant genera across all groups were Prevotella_1 
A

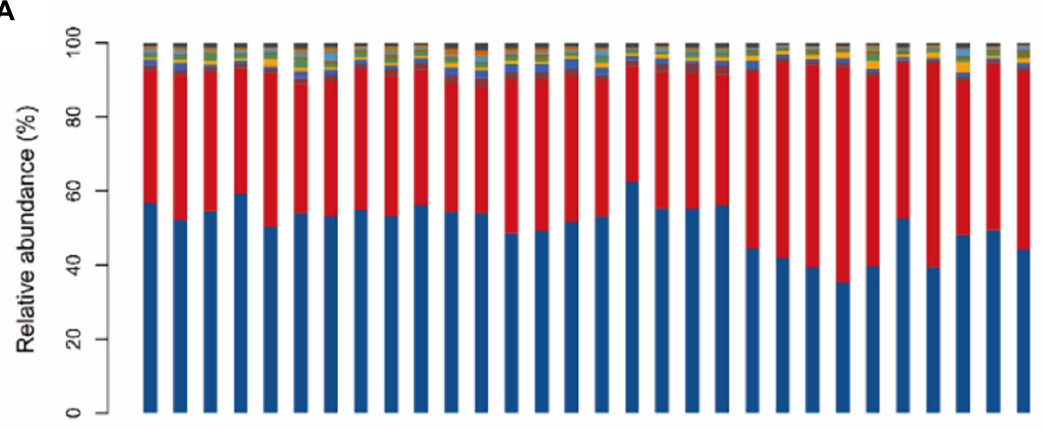

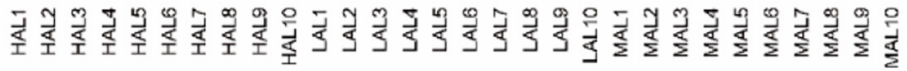

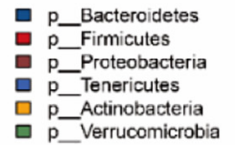

P__Saccharibacteria
P_SR1_Absconditabacteria

P_ P _ Synergistetes

ㅁ. P.Cyanobacteria

B

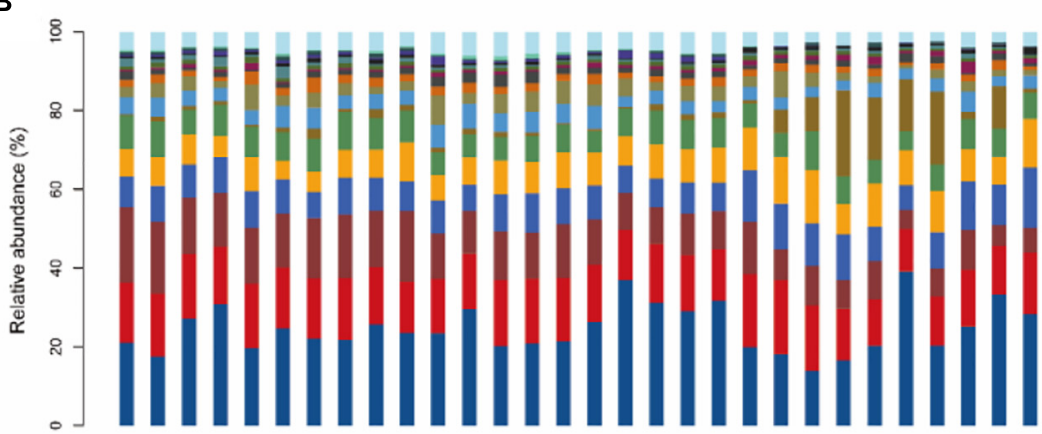

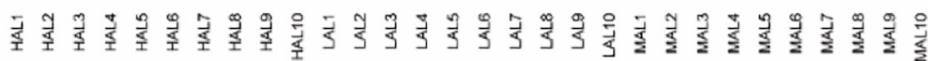

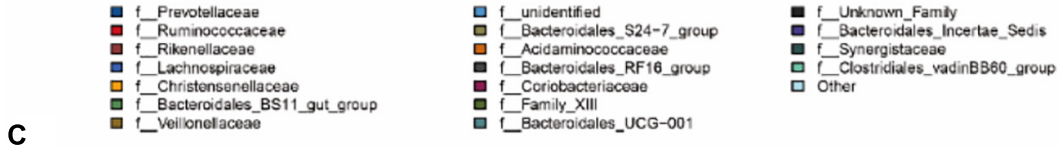

C

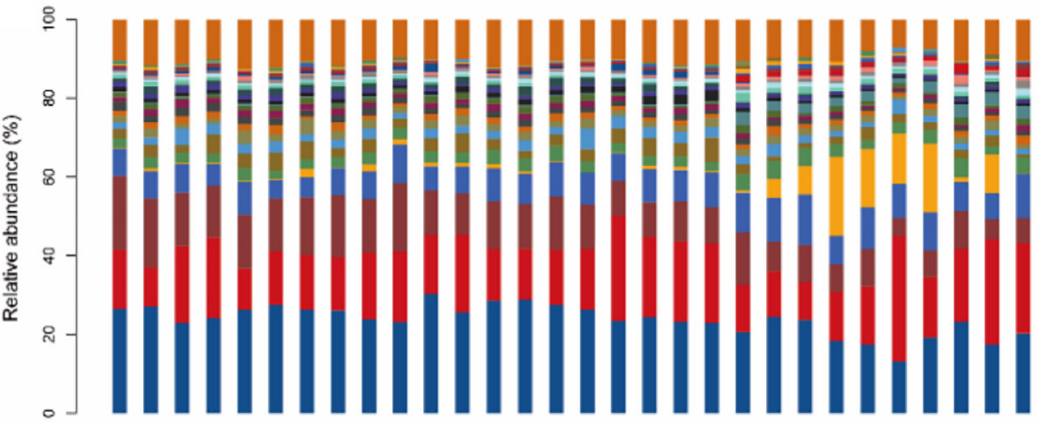

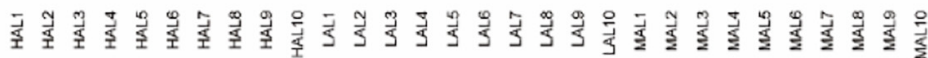
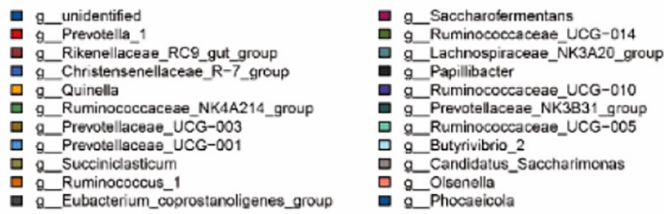

E g_Acetitomaculum

g_Ruminococcus_2 2 9PB1014_grou

g_Lachnospirace

口. Ruminococcaceae UCG-C01

므_unidentified_rumen_bacterium_RFN4

g_Eubacterium_ventriosum_group

- Eubacterium coprostanoligenes group - 2- Phocaeicola

FIGURE 2 | Compositional profiles of rumen microbiota from yaks inhabiting regions located at distinct altitudes. (A) Phylum level, (B) family level, and (C) genus level. HAL, high-altitude region (Zhongba County, Xigatse City; 4,800 m); MAL, medium-altitude region (Nagqu City; 4,500 m); LAL, low-altitude region (Dangxiong County, Lhasa City; $3,800 \mathrm{~m}$ ). 

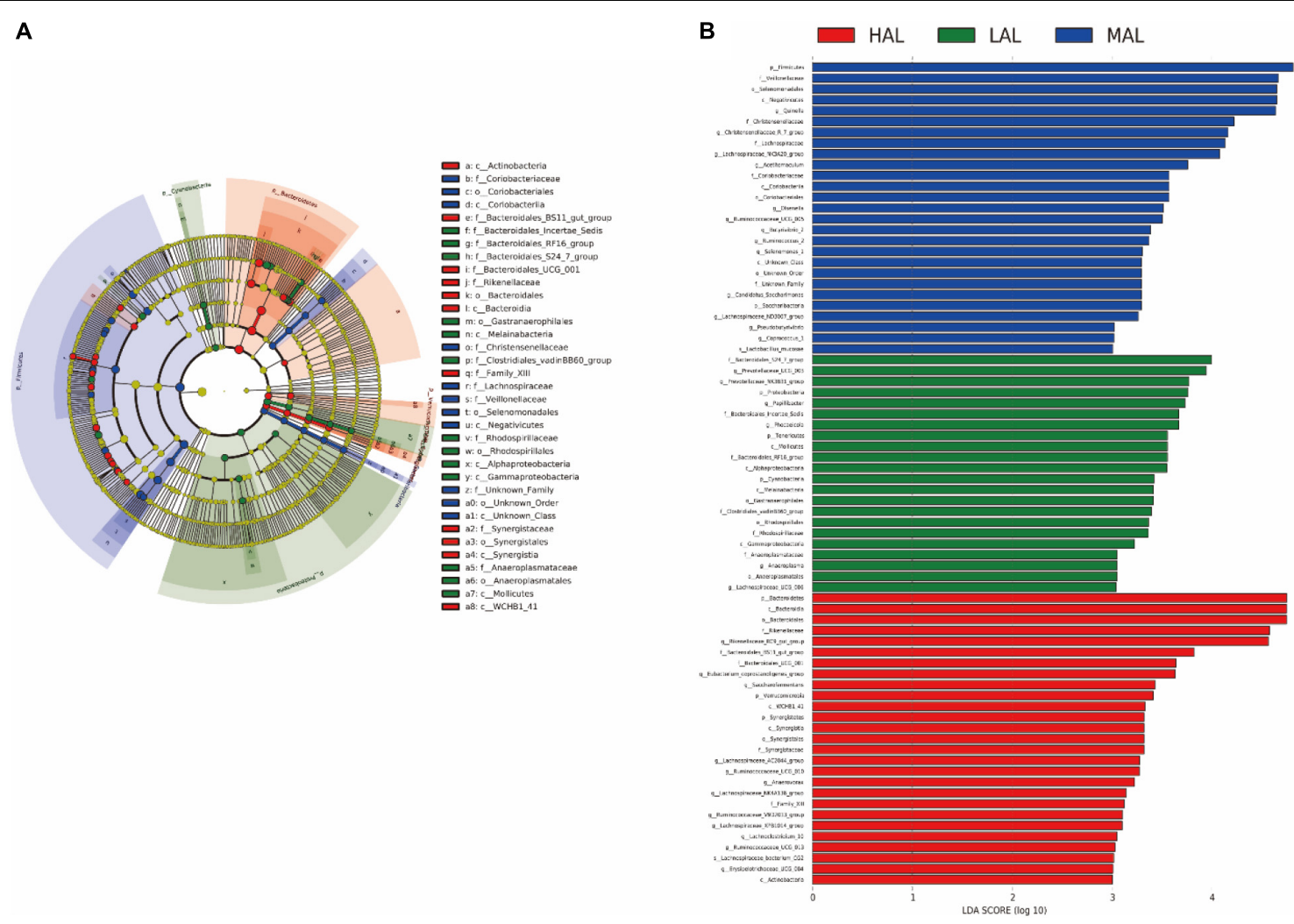

FIGURE 3 | LEfSe analysis of rumen microbiota from yaks inhabiting regions located at distinct altitudes. (A) Histogram of linear discriminant analysis scores based on Classification Information. (B) Linear discriminant analysis effect size cladogram based on Classification Information. HAL, high-altitude region (Zhongba County, Xigatse City; 4,800 m); MAL, medium-altitude region (Nagqu City; 4,500 m); LAL, low-altitude region (Dangxiong County, Lhasa City; 3,800 m).

(16.82\%), Rikenellaceae_RC9_gut_group (11.26\%), and Christensenellaceae_R-7_group (8.06\%) (Figure 2C and Supplementary Table 3). Specifically, the MAL group displayed the highest $(p<0.05)$ relative abundances of Christensenellaceae_R-7_group, Ruminococcaceae_NK4A214 group, Quinella, Ruminococcaceae_UCG-005, Olsenella, Butyrivibrio_2, Lachnospiraceae_NK3A20_group, and Acetitomaculum, but the lowest $(p<0.05)$ relative abundances of Prevotellaceae_UCG-003, Rikenellaceae_RC9_gut_group, Eubacterium_coprostanoligenes_group, Saccharofermentans, and Prevotellaceae_NK3B31_group compared with the LAL and HAL groups. The relative abundance of Ruminococcaceae_UCG-010 was significantly $(p<0.05)$ higher in the HAL group compared with the MAL and LAL groups, whereas the relative abundance of Papillibacter was higher $(p<0.05)$ in the LAL group than in the other groups.

To better understand the dominance of specific bacteria within the three groups, we used the LEfSe method (Figure 3). The Firmicutes and Saccharibacteria phyla, including genera Quinella, Christensenellaceae_R_7_group, Lachnospiraceae _NK3A20_group, Acetitomaculum, Olsenella, Ruminococcaceae _UCG_005, Butyrivibrio_2, and Ruminococcus_2, were abundant in the MAL group. The Bacteroidetes, Verrucomicrobia, and Synergistetes phyla, including Rikenellaceae_RC9_gut _group, Saccharofermentans, Lachnospiraceae_AC2044_group,
Ruminococcaceae_UCG_010, Anaerovorax, and Family_XIII, were enriched in the HAL group. Finally, the Papillibacter, Tenericutes, Cyanobacteria, and Bacteroidales_S24_7_group phyla, including Prevotellaceae_UCG_003, Prevotellaceae_NK3 B31_group, Papillibacter, Anaeroplasma, and Lachnospiraceae _UCG_006, were over-represented in the LAL group.

\section{Rumen Fermentation Parameters and Altitude Correlate With Bacterial Communities}

The correlation between relative abundance of the top 20 bacterial genera, fermentation parameters, and altitude (Figure 4A) revealed that the relative abundances of Rikenellaceae_RC9_gut_group, Quinella, Prevotellaceae_UCG003, Lachnospiraceae_NK3A20_group, Papillibacter, Ruminococcaceae_UCG-010, Prevotellaceae_NK3B31_group, and Ruminococcaceae_UCG-005 correlated with altitude and rumen fermentation $(p<0.05)$. For example, the relative abundance of Rikenellaceae_RC9_gut_group correlated positively with altitude $(r=0.84)$ and A/P $(r=0.47)$, whereas the relative abundance of Quinella correlated negatively with altitude $(r=-0.49)$ and butyric acid $(r=-0.43)$. The relative abundance of Papillibacter correlated positively with altitude $(r=0.41)$, total VFA $(r=0.62)$, propionic acid $(r=0.45)$, and butyric acid $(r=0.62)$, but 
A

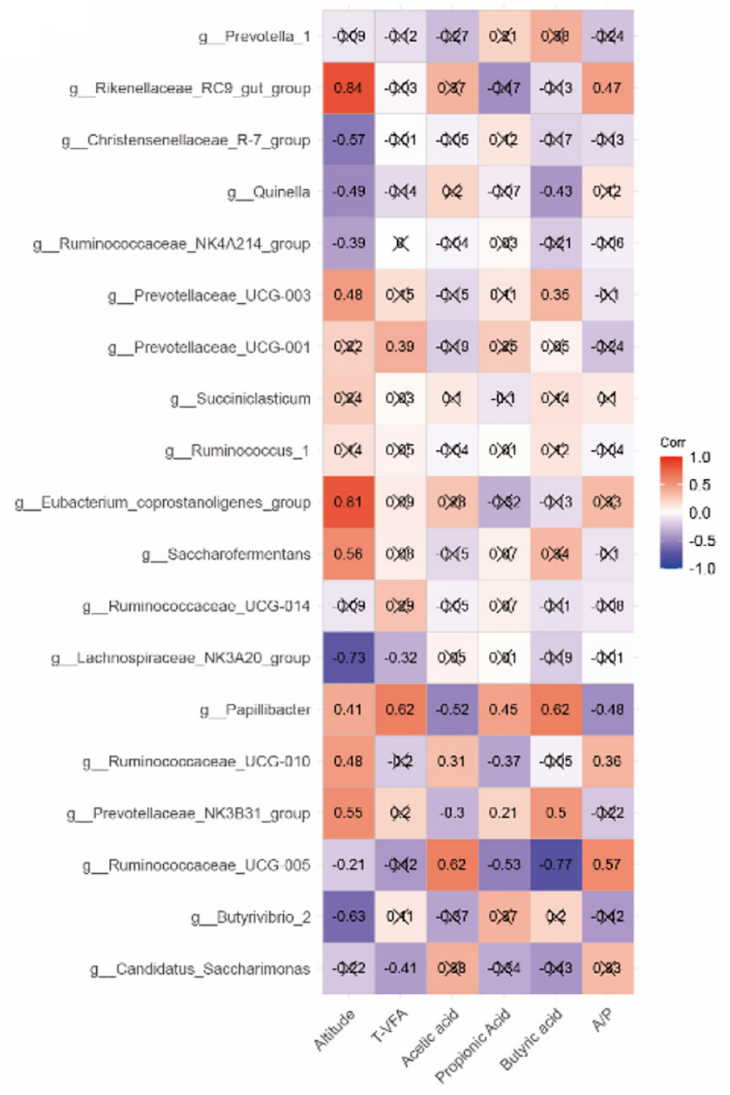

B

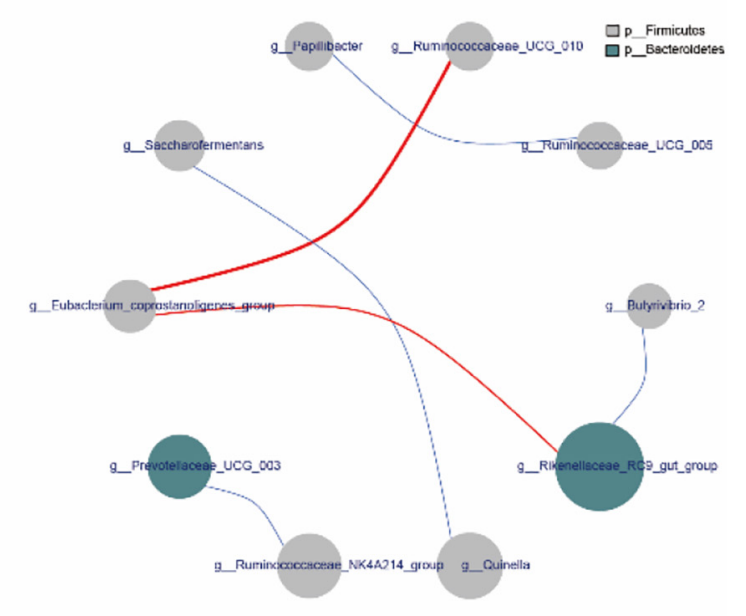

FIGURE 4 | Correlation analysis of rumen genera in yaks inhabiting regions located at distinct altitudes. (A) Correlation between the top 20 relative abundances of genera, fermentation parameters, and altitude. (B) Interaction among the top 20 relative abundances at genus level. Red represents a positive correlation and blue a negative correlation. The number represents the correlation coefficient. The $\times$ sign represents $p>0.05$. The size and color of the circle represent the relative abundance of the respective genus and the phylum.

negatively with acetic acid $(r=-0.52)$ and A/P $(r=-0.48)$. Interestingly, we found a significant negative correlation between Papillibacter and Ruminococcaceae_UCG-005 $(|r|>0.6$, $p<0.05$; Figure 4B).

\section{PICRUSt2 Function Prediction}

The function of rumen microbial communities in yaks from three different regions was predicted by PICRUSt2 software and related to KEGG pathways (Table 3). Notably, "Biosynthesis of ansamycins" (4.47\%) was the most abundant pathway in all three groups, followed by "Biosynthesis of vancomycin group antibiotics" (2.47\%), "Valine, leucine and isoleucine biosynthesis" $(2.25 \%)$, and "D-Glutamine and D-glutamate metabolism" (2.19\%). The Kruskal-Wallis test confirmed that a total of 25 pathways showed significant $(p<0.05)$ differences among the three groups (Table 3). Specifically, "Amino acid metabolism," "D-Alanine metabolism," and "Thiamine metabolism" pathways, as well as "Carbohydrate metabolism," "Cell motility", and "Folding, sorting and degradation" gene categories were significantly $(p<0.05)$ more abundant in the MAL than in the other two groups.

\section{DISCUSSION}

This is the first study to evaluate the influence of extreme environments, corresponding to different altitudes of the Tibetan Plateau, on rumen microbiota in yaks. The study highlights how rumen fermentation, bacterial composition, and function are related to the regions in which the yaks live.

Ruminants rely on VFAs produced in the rumen during fermentation as a source of energy. Indeed, their feed efficiency is related to such VFAs (Lam et al., 2018; Zhou et al., 2018). While propionic acid can supply additional energy to ruminants, acetic and butyric acids are converted to methane and carbon dioxide during the consumption process, lowering energy efficiency (Gunun et al., 2018). Propionic acid and butyric acid compete with methane for hydrogen during fermentation, reducing methane energy consumption while improving energy supply (Pragna et al., 2018). Acetic acid and butyric acid originate mainly from the fermentation of fiber, whereas propionic acid derives from the fermentation of sugar and starch (Astawa et al., 2011). In this study, VFAs and propionic acid were highest in the rumen of MAL animals, suggesting that feed efficiency might be better in this than in the other groups. 
TABLE 3 | Functional predictions for rumen microbiota in yaks inhabiting regions located at distinct altitudes; only significantly different KEGG pathways are shown.

\begin{tabular}{|c|c|c|c|c|c|}
\hline KEGG_Pathways & \multicolumn{3}{|c|}{ Groups $^{1}$} & \multirow[t]{2}{*}{ SEM } & \multirow[t]{2}{*}{$P$-value } \\
\hline Level III & HAL & MAL & LAL & & \\
\hline \multicolumn{6}{|l|}{ Metabolism } \\
\hline \multicolumn{6}{|l|}{ Metabolism of terpenoids and polyketides } \\
\hline Biosynthesis of ansamycins & $4.51^{a}$ & $4.63^{a}$ & $4.26^{b}$ & 0.052 & 0.0113 \\
\hline Biosynthesis of vancomycin group antibiotics & $2.53^{a}$ & $2.37^{b}$ & $2.50^{a}$ & 0.015 & $<0.001$ \\
\hline Terpenoid backbone biosynthesis & $1.40^{b}$ & $1.42^{a}$ & $1.40^{b}$ & 0.002 & 0.0112 \\
\hline \multicolumn{6}{|l|}{ Amino acid metabolism } \\
\hline Valine, leucine, and isoleucine biosynthesis & $2.23^{b}$ & $2.29^{a}$ & $2.22^{b}$ & 0.007 & $<0.001$ \\
\hline Lysine biosynthesis & $1.52^{b}$ & $1.59^{a}$ & $1.53^{b}$ & 0.003 & $<0.001$ \\
\hline Phenylalanine, tyrosine, and tryptophan biosynthesis & $1.28^{b}$ & $1.33^{a}$ & $1.29^{b}$ & 0.004 & $<0.001$ \\
\hline Histidine metabolism & $1.26^{a}$ & $1.26^{a}$ & $1.25^{b}$ & 0.002 & 0.0012 \\
\hline Cysteine and methionine metabolism & $1.22^{b}$ & $1.28^{a}$ & $1.23^{b}$ & 0.005 & $<0.001$ \\
\hline \multicolumn{6}{|l|}{ Metabolism of other amino acids } \\
\hline D-Glutamine and D-glutamate metabolism & $2.17^{b}$ & $2.21^{a}$ & $2.18^{a b}$ & 0.006 & 0.0393 \\
\hline D-Alanine metabolism & $1.60^{b}$ & $1.65^{a}$ & $1.60^{b}$ & 0.006 & $<0.001$ \\
\hline Selenocompound metabolism & $1.06^{a}$ & $1.02^{C}$ & $1.03^{b}$ & 0.004 & $<0.001$ \\
\hline \multicolumn{6}{|l|}{ Metabolism of cofactors and vitamins } \\
\hline Pantothenate and CoA biosynthesis & $1.86^{b}$ & $1.90^{a}$ & $1.86^{b}$ & 0.006 & 0.0125 \\
\hline Thiamine metabolism & $1.50^{b}$ & $1.60^{a}$ & $1.53^{b}$ & 0.006 & 0.0016 \\
\hline Folate biosynthesis & $1.25^{a}$ & $1.15^{b}$ & $1.24^{a}$ & 0.011 & $<0.001$ \\
\hline Nicotinate and nicotinamide metabolism & $1.12^{b}$ & $1.11^{b}$ & $1.14^{a}$ & 0.005 & 0.0178 \\
\hline Lipoic acid metabolism & $1.12^{b}$ & $1.03^{b}$ & $1.18^{a}$ & 0.020 & 0.0195 \\
\hline \multicolumn{6}{|l|}{ Carbohydrate metabolism } \\
\hline C5-Branched dibasic acid metabolism & $1.69^{b}$ & $1.80^{a}$ & $1.68^{b}$ & 0.015 & 0.0063 \\
\hline Pentose phosphate pathway & $1.52^{b}$ & $1.57^{a}$ & $1.51^{b}$ & 0.010 & 0.0015 \\
\hline Citrate cycle (TCA cycle) & $1.17^{a}$ & $1.10^{c}$ & $1.15^{b}$ & 0.006 & $<0.0001$ \\
\hline Amino sugar and nucleotide sugar metabolism & $1.03^{b}$ & $1.07^{a}$ & $1.04^{b}$ & 0.004 & 0.0145 \\
\hline \multicolumn{6}{|l|}{ Lipid metabolism } \\
\hline Fatty acid biosynthesis & $1.74^{a}$ & $1.65^{b}$ & $1.69^{b}$ & 0.011 & 0.0153 \\
\hline \multicolumn{6}{|l|}{ Energy metabolism } \\
\hline Carbon fixation pathways in prokaryotes & $1.29^{a}$ & $1.25^{b}$ & $1.27^{b}$ & 0.004 & $<0.001$ \\
\hline \multicolumn{6}{|l|}{ Cellular processes } \\
\hline \multicolumn{6}{|l|}{ Cell motility } \\
\hline Bacterial chemotaxis & $1.112^{b}$ & $1.505^{a}$ & $1.130^{b}$ & 0.039 & $<0.001$ \\
\hline \multicolumn{6}{|l|}{ Genetic information processing } \\
\hline \multicolumn{6}{|l|}{ Folding, sorting, and degradation } \\
\hline Protein export & $1.486^{b}$ & $1.503^{a}$ & $1.485^{b}$ & 0.007 & 0.0032 \\
\hline \multicolumn{6}{|l|}{ Replication and repair } \\
\hline DNA replication & $1.279^{a}$ & $1.263^{b}$ & $1.272^{a b}$ & 0.002 & 0.0249 \\
\hline
\end{tabular}

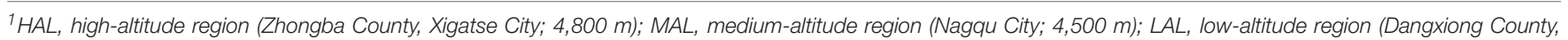
Lhasa City; 3,800 m).

Different superscript letters in the same row denote significant differences $(p<0.05)$.

Contrary to the present results, Zhang et al. (2016) found that ruminants living at elevated altitude had higher VFA content and significantly upregulated genes responsible for VFA absorption in the rumen. Diet is the most important factor regulating development and colonization of rumen microbiota (Zhou et al., 2018; Lin et al., 2019; Myer, 2019). Ram (2005) reported that meadows found at different elevation in the Uttaranchal Himalaya exhibited distinct species richness. Similarly, Bao et al. (2021) linked the alpine meadows between Dangxiong and Nagqu regions of Tibet with a varied nutritional value.
Regional variation could be related to temperature differences between day and night, photosynthesis, and topography (Han et al., 1997; Skapetas et al., 2004). Accordingly, further experiments are required to ascertain whether the observed differences are due to the extreme climatic conditions and altitude tested in this study or to the quality of grass in the different regions. Furthermore, the alpha and beta diversity indices of rumen microbiota differed significantly between the three groups, indicating a close relationship between the diversity of rumen microbiota in yaks and geographic location. 
Interestingly, unlike VFAs, the diversity of ruminal microbiota first decreased and then increased with elevation. Shabat et al. (2016) found that microbiota diversity correlated negatively with feed efficiency, with efficient dairy cattle exhibiting lower rumen microbiota diversity, methane emission, and acetic acid concentration, but higher propionic acid content. Taking into account those and the present results, it appears that yaks in the MAL group are more efficient at utilizing their pasture and emit less methane.

As observed previously (Zhang et al., 2016; Guo et al., 2021; Liu et al., 2021), Bacteroidetes and Firmicutes were the dominant taxa identified in this study. Guo et al. (2020) reported that Bacteroidetes were the most important bacteria in yak rumen from birth to adulthood. Here, we found that Firmicutes were the signature bacteria of the MAL group as they were significantly more abundant than in the other two groups. Because Firmicutes play an important role in energy conversion (Turnbaugh et al., 2006), our results indicated that they might be the key enablers of high feed utilization by yaks in the MAL group. Furthermore, the relative abundance of Papillibacter was found to correlate positively with altitude, whereas Ruminococcaceae_UCG_005 showed a negative correlation. Mao et al. (2013) found that dairy cattle had lower levels of Papillibacter when in a state of subacute ruminal acidosis, which indirectly suggests that yaks may be better suited to living at higher altitudes. Ruminococcaceae_UCG_005 enterotype has been associated with yak diets rich in proteins but low in fibers (Guo et al., 2021). Combined with the correlation results of VFAs, we hypothesize that Papillibacter and Ruminococcaceae_UCG_005 may be the hub genera allowing for adaptation to high altitude. By antagonizing each other, they may allow yaks to adjust to different altitudes through modulation of the ruminal VFA content. The genera Rikenellaceae_RC9_gut_group, Quinella, Prevotellaceae_UCG-003, Lachnospiraceae_NK3A20_group, Prevotellaceae_NK3B31_group, and Ruminococcaceae_UCG-005 exhibited a correlation with different altitudes and rumen fermentation parameters, suggesting that they, too, may play a key part in the adaptation to extreme environments. As there are other reports pointing to the involvement of altitude-related genera in VFA production, rumen development, immunity, and methane output (Petri et al., 2013; Zhai et al., 2017; Pragna et al., 2018; Lin et al., 2019; Sha et al., 2021), we believe that altitude affects the microbial composition of yaks' rumen and enables adaptation to extreme environments by modulating rumen fermentation products.

The functional properties of microbiota dictate the hostmicrobiome interaction (Lin et al., 2019; Myer, 2019). Here, the PICRUSt2 result revealed that the function of yak rumen microbiota differed between the three regions. In contrast, Liu et al. (2021) found a similar functional gene composition of yak intestinal microbes across the three regions, suggesting that the yak rumen may be more sensitive than the intestinal tract in terms of environmental adaptability. Due to limited experimental conditions, this study did not further explore how diet and extreme environment affected microbiota composition beyond the correlation with altitude.
The mechanism regulating adaptation between yak genome and extreme environment has been described previously (Qiu et al., 2012), and the microbiome is closely related to animal activities ( $\mathrm{Wu}$ et al., 2011). Therefore, future metagenomics studies may help untangle the contribution of gut microorganisms to mammalian adaptation to extreme environments.

\section{CONCLUSION}

This study has analyzed the rumen microbiota and fermentation products of yaks living at different altitudes in Tibet, China. Indeed, rumen fermentation, composition, and function were found to vary across regions, with some genera correlating strongly with altitude. Based on the results, we found the middle-altitude region was suitable for rumen fermentation endproducts and microbiota composition compared with the low- or high-altitude regions in Tibet. These results provide an insight on the mechanism enabling yaks to adapt to high altitude and maximize feed efficiency in extreme environments.

\section{DATA AVAILABILITY STATEMENT}

The datasets presented in this study can be found in online repositories. The names of the repository/repositories and accession number(s) can be found below: https://submit.ncbi. nlm.nih.gov/subs/, PRJNA776716.

\section{ETHICS STATEMENT}

The animal study was reviewed and approved by the Chinese Academy of Agricultural Sciences Animal Ethics Committee approved the experimental protocol, and all the methods conducted in this experiment were in accordance with humane animal care and handling procedures (AEC-CAAS-20190905). Written informed consent was obtained from the owners for the participation of their animals in this study.

\section{AUTHOR CONTRIBUTIONS}

WX: conceptualization. KC: methodology, supervision, and funding acquisition. LH: software, resources, data curation, and writing-original draft preparation. YT, QD, and XC: validation. $\mathrm{HC}$ : formal analysis and visualization. FQ: investigation. LH, WX, TM, KC, and CZ: writing-review and editing. KC and CZ: project administration. All authors contributed to the article and approved the submitted version.

\section{FUNDING}

This research was funded by the State Key Laboratory of Hulless Barley and Yak Germplasm Resources and Genetic Improvement (XZNKY-2020-C-007K02). The Key R\&D Program of Tibet Autonomous Region (XZ201901NB01). 


\section{ACKNOWLEDGMENTS}

We thank Shuo Wang from the College of Animal Science and Technology, China Agricultural University, for his guidance on the article.

\section{REFERENCES}

Anantasook, N., Wanapat, M., Cherdthong, A., and Gunun, P. (2013). Changes of microbial population in the rumen of dairy steers as influenced by plant containing tannins and saponins and roughage to concentrate ratio. Asian Australas J. Anim. Sci. 26, 1583-1591. doi: 10.5713/ajas.2013.13182

Astawa, P. A., Partama, I., Suyadnya, P., and Sutarpa, I. (2011). Effect of vitamin - mineral supplementation in commercial feed on the digestibility coefficient and rumen fermentation of bali cattle. J. Indones Trop Anim. 36, 69-74. doi: 10.14710/jitaa.36.1.69-74

Bao, Y. H., Wang, W. B., Yuan, X. J., Pubu, Z. M., Luo, Z., and Can, M. Y. (2021). Comparative study on nutritive value of Kobresia tibetica of alpine meadow in dangxiong and naqu regions of qinghai-tibet plateau. Chin. J. Anim. Nutr. 33, 6233-6244. doi: 10.3969/j.issn.1006-267x.2021.11.024

Bi, Y. L., Tu, Y., Zhang, N. F., Wang, S. Q., Zhang, F., Suen, G., et al. (2021). Multiomics analysis reveals the presence of a microbiome in the gut of fetal lambs. Gut 70, 853-864. doi: 10.1136/gutjnl-2020-320951

Bolger, A. M., Lohse, M., and Usadel, B. (2014). Trimmomatic: a flexible trimmer for illumina sequence data. Bioinformatics 30, 2114-2120. doi: 10.1093/ bioinformatics/btu170

Douglas, G. M., Maffei, V. J., Zaneveld, J., Yurgel, S. N., and Langille, M. (2019) PICRUSt2: an improved and extensible approach for metagenome inference. BioRxiv [preprint]. *BioRxiv. 672295, doi: 10.1101/672295

Edgar, R. C. (2013). UPARSE: highly accurate OTU sequences from microbial amplicon reads. Nat. Methods 10, 996-998. doi: 10.1038/nmeth.2604

Furman, O., Shenhav, L., Sasson, G., Kokou, F., Honig, H., Jacoby, S., et al. (2020). Stochasticity constrained by deterministic effects of diet and age drive rumen microbiome assembly dynamics. Nat. Commun. 11:1904. doi: 10.1038/s41467020-15652-8

Gao, J., Liu, M., and Meng, X. (2013). Seroprevalence of bovine viral diarrhea infection in yaks (bos grunniens) on the qinghai-tibetan plateau of China. Trop Anim. Health Pro. 45, 791-793. doi: 10.1007/s11250-012-0290-2

Guan, L. L., Nkrumah, J. D., Basarab, J. A., and Moore, S. S. (2008). Linkage of microbial ecology to phenotype: correlation of rumen microbial ecology to cattle's feed efficiency. FEMS Microbiol. Lett. 288, 85-91. doi: 10.1111/j.15746968.2008.01343.x

Gunun, P., Gunun, N., Cherdthong, A., Wanapat, M., Polyorach, S., Sirilaophaisan, S., et al. (2018). In vitro rumen fermentation and methane production as affected by rambutan peel powder. J. Appl. Anim. Res. 46, 626-631. doi: 10.1080/ 09712119.2017 .1371608

Guo, N., Wu, Q., Shi, F., Niu, J., Zhang, T., Degen, A. A., et al. (2021). Seasonal dynamics of diet-gut microbiota interaction in adaptation of yaks to life at high altitude. NPJ Biofilms Microbiomes. 7:38. doi: 10.1038/s41522-021-00207-6

Guo, W., Zhou, M., Ma, T., Bi, S., Wang, W., Zhang, Y., et al. (2020). Survey of rumen microbiota of domestic grazing yak during different growth stages revealed novel maturation patterns of four key microbial groups and their dynamic interactions. Anim Microbiome. 2, 23. doi: 10.1186/s42523-02000042-8

Guo, X., Pei, J., Bao, P. J., Chu, M., Wu, X. Y., Ding, X. Z., et al. (2015). The complete mitochondrial genome of the qinghai plateau yak bos grunniens (cetartiodactyla: bovidae). Mitochondrial DNA A DNA Mapp. Seq. Anal. 27, 2889-2890. doi: 10.3109/19401736.2015.1060423

Han, F., Chen, G. Y., and Shi, S. B. (1997). Contents of protein, fat and starch of kobresia humilis plants grown at different altitude in qinghai-xizang plateau. Acta Ecol. Sin. 21, 97-104.

Khan, M. A., Bach, A., Weary, D. M., and von Keyserlingk, M. A. G. (2016). Invited review: transitioning from milk to solid feed in dairy heifers. J. Dairy Sci. 99, 885-902. doi: 10.3168/jds.2015-9975

Knight, R. (2011). UCHIME improves sensitivity and speed of chimera detection. Bioinformatics 27, 2194-2200. doi: 10.1093/bioinformatics/btr381

\section{SUPPLEMENTARY MATERIAL}

The Supplementary Material for this article can be found online at: https://www.frontiersin.org/articles/10.3389/fmicb. 2021.807512/full\#supplementary-material

Lam, S., Munro, J. C., Zhou, M., Guan, L. L., Schenkel, F. S., Steele, M. A., et al. (2018). Associations of rumen parameters with feed efficiency and sampling routine in beef cattle. Animal 12, 1442-1450. doi: 10.1017/S1751731117002750

Li, K., Mehmood, K., Zhang, H., Jiang, X., Shahzad, M., Dong, X., et al. (2018). Characterization of fungus microbial diversity in healthy and diarrheal yaks in gannan region of tibet autonomous prefecture. Acta Tropica 182, 14-26. doi: 10.1016/j.actatropica.2018.02.017

Liang, Y. S., Li, G. Z., Li, X. Y., Lv, J. Y., Li, F. D., Tang, D. F., et al. (2017). Growth performance, rumen fermentation, bacteria composition, and gene expressions involved in intracellular $\mathrm{pH}$ regulation of rumen epithelium in finishing hu lambs differing in residual feed intake phenotype. J. Anim. Sci. 95, 1727-2738. doi: $10.2527 /$ jas.2016.1134

Lin, L., Xie, F., Sun, D. M., Liu, J. H., Zhu, W. Y., and Mao, S. Y. (2019). Ruminal microbiome-host crosstalk stimulates the development of the ruminal epithelium in a lamb model. Microbiome 7:83. doi: 10.1186/s40168-019-0701-y

Liu, C., Wu, H., Liu, S., Chai, S., Meng, Q., and Zhou, Z. (2019). Dynamic alterations in yak rumen bacteria community and metabolome characteristics in response to feed type. Front. Microbiol. 10:1116. doi: 10.3389/fmicb.2019.0 1116

Liu, J., Cai, J. Z., Zhang, W., Chen, D., and Liu, Q. R. (2008). Seroepidemiology of neospora caninum and toxoplasma gondii infection in yaks (bos grunniens) in qinghai, china. Vet. Parasitol. 152, 330-332. doi: 10.1016/j.vetpar.2007.12.010

Liu, W. W., Wang, Q., Song, J. J., Xin, J. W., Zhang, S. S., Lei, Y. H., et al. (2021). Comparison of gut microbiota of yaks from different geographical regions. Front. Microbiol. 12:666940. doi: 10.3389/fmicb.2021.666940

Long, R. J., Ding, L. M., Shang, Z. H., and Guo, X. H. (2008). The yak grazing system on the qinghai-tibetan plateau and its status. Rangeland J. 30, 241-246.

Mago, T., and Salzberg, S. L. (2011). FLASH: fast length adjustment of short reads to improve genome assemblies. Bioinformatics 27, 2957-2963. doi: 10.1093/ bioinformatics/btr507

Mao, S. Y., Zhang, R. Y., Wang, D. S., and Zhu, W. Y. (2013). Impact of subacute ruminal acidosis (SARA) adaptation on rumen microbiota in dairy cattle using pyrosequencing. Anaerobe 24, 12-19. doi: 10.1016/j.anaerobe.2013.08.003

Miller, G. E., Engen, P. A., Gillevet, P. M., Maliha, S., Masoumeh, S., Forsyth, C. B., et al. (2016). Lower neighborhood socioeconomic status associated with reduced diversity of the colonic microbiota in healthy adults. PLoS One 11:e148952. doi: 10.1371/journal.pone.0148952

Myer, P. R. (2019). Bovine genome-microbiome interactions: metagenomic frontier for the selection of efficient productivity in cattle systems. mSystems 4, e103-e119. doi: 10.1128/mSystems.00103-19

O'Hara, E., Neves, A. L. A., Song, Y., and Guan, L. L. (2020). The role of the gut microbiome in cattle production and health: driver or passenger? Annu Rev. Anim Biosci. 8, 199-220. doi: 10.1146/annurev-animal-021419-083952

Oliveira, A. C., Rebelo, A. R., and Homem, C. (2021). Integrating animal development: how hormones and metabolism regulate developmental transitions and brain formation. Dev. Biol. 475, 256-264. doi: 10.1016/j.ydbio.2021.01.016

Petri, R. M., Schwaiger, T., Penner, G. B., Beauchemin, K. A., Forster, R. J., Mckinnon, J. J., et al. (2013). Changes in the rumen epimural bacterial diversity of beef cattle as affected by diet and induced ruminal acidosis. Appl. Environ. Microb. 79, 3744-3755. doi: 10.1128/AEM.03983-12

Pragna, P., Chauhan, S. S., Sejian, V., Leury, B. J., and Dunshea, F. R. (2018). Climate change and goat production: enteric methane emission and its mitigation. Animals 8:235. doi: 10.3390/ani8120235

Qiu, Q., Zhang, G., Auvil, L., Capitanu, B., Ma, J., Lewin, H. A., et al. (2012). The yak genome and adaptation to life at high altitude. Nat. Genet. 44, 946-949. doi: 10.1038/ng.2343

Quast, C., Pruesse, E., Yilmaz, P., Gerken, J., and Glckner, F. O. (2012). The SILVA ribosomal RNA gene database project: improved data processing and web-based tools. Nucleic Acids Res. 41, D590-D596. doi: 10.1093/nar/gks1219 
Ram, J. (2005). Biodiversity and conservation of high altitude meadows of uttaranchal himalaya. Bull. Natl. Inst. Ecol. 15, 11-17.

Ramos-Morales, E., Arco-Pérez, A., Martín-García, A. I., Ez-Ruiz, D. Y. Frutos, P., and Hervás, G. (2014). Use of stomach tubing as an alternative to rumen cannulation to study ruminal fermentation and microbiota in sheep and goats. Anim Feed Sci. Tech. 198, 57-66. doi: 10.1016/j.anifeedsci.2014. 09.016

Sha, Y. Z., Hu, J., Shi, B. G., Dingkao, R. Q., Wang, J. Q., Li, S. B., et al. (2021). Supplementary feeding of cattle-yak in the cold season alters rumen microbes, volatile fatty acids, and expression of SGLT1 in the rumen epithelium. PeerJ. 9:e11048. doi: 10.7717/peerj.11048

Shabat, S., Sasson, G., Doron-Faigenboim, A., Durman, T., Yaacoby, S., Miller, M. B., et al. (2016). Specific microbiome-dependent mechanisms underlie the energy harvest efficiency of ruminants. ISME J. 10, 2958-2972. doi: 10.1038/ ismej.2016.62

Skapetas, B., Nitas, D., Karalazos, A., and Hatziminaoglou, I. (2004). A study on the herbage mass production and quality for organic grazing sheep in a mountain pasture of northern Greece. Livest Prod. Sci. 87, 277-281. doi: 10. 1016/j.livprodsci.2003.08.002

Takahashi, S., Tomita, J., Nishioka, K., Hisada, T., Nishijima, M., and Bourtzis, K. (2014). Development of a prokaryotic universal primer for simultaneous analysis of bacteria and archaea using next-generation sequencing. PLoS One 9:e105592. doi: 10.1371/journal.pone.0105592

Turnbaugh, P. J., Ley, R. E., Mahowald, M. A., Magrini, V., Mardis, E. R., and Gordon, J. I. (2006). An obesity-associated gut microbiome with increased capacity for energy harvest. Nature 444, 1027-1031. doi: 10.1038/nature0 5414

Wang, Q. (2007). Naive bayesian classifier for rapid assignment of rRNA sequences into the new bacterial taxonomy. Appl. Environ. Microbiol. 73, 5261-5267. doi: 10.1128/AEM.00062-07

Whiteley, N. M., and Faulkner, L. S. (2005). Temperature influences whole-animal rates of metabolism but not protein synthesis in a temperate intertidal isopod. Physiol. Biochem Zool. 78, 227-238. doi: 10.1086/427054

Wu, D. W., Vinitchaikul, P., Deng, M. Y., Zhang, G. R., Sun, L. Y., Wang, H. X., et al. (2021). Exploration of the effects of altitude change on bacteria and fungi in the rumen of yak (bos grunniens). Arch. Microbiol. 203, 1-12. doi: 10.1007/s00203-020-02072-x

Wu, G. D., Chen, J., Hoffmann, C., Chen, Y. Y., Keilbaugh, S. A., Bewtra, M., et al. (2011). Linking long-term dietary patterns with gut microbial enterotypes. Science 334, 105-108. doi: 10.1126/science.1208344
Yue, Q., Yang, H. J., Cao, Y. C., Zhang, D. F., Jiang, Y. H., and Wang, J. Q. (2009). Feruloyl and acetyl esterase production of an anaerobic rumen fungus Neocallimastix sp. YQ2 effected by glucose and soluble nitrogen supplementations and its potential in the hydrolysis of fibrous feedstuffs. Anim. Feed Sci. Tech. 153, 263-277. doi: 10.1016/j.anifeedsci.2009.06.019

Zhai, Q. X., Li, T. Q., Yu, L. L., Xiao, Y., Feng, S. S., Wu, J. P., et al. (2017). Effects of subchronic oral toxic metal exposure on the intestinal microbiota of mice. Sci. Bull. 62, 831-840. doi: 10.1016/j.scib.2017.01.031

Zhang, J., Yang, M., Cai, D. Y. C., Hao, Y. J., Zhao, X., Zhu, Y. H., et al. (2020). Composition, coagulation characteristics, and cheese making capacity of yak milk. J. Dairy Sci. 103, 1276-1288. doi: 10.3168/jds.2019-17231

Zhang, Y., Li, F., Chen, Y., Wu, H., Meng, Q., and Guan, L. L. (2020) Metatranscriptomic profiling reveals the effect of breed on active rumen eukaryotic composition in beef cattle with varied feed efficiency. Front. Microbiol. 11:367. doi: 10.3389/fmicb.2020.00367

Zhang, Z. G., Xu, D. M., Wang, L., Hao, J. J., Wang, J. F., Zhou, X., et al. (2016). Convergent evolution of rumen microbiomes in high-altitude mammals. Curr. Biol. 26, 1873-1879. doi: 10.1016/j.cub.2016.05.012

Zhou, M., Peng, Y. J., Chen, Y. H., Klinger, C. M., Oba, M., Liu, J. X., et al. (2018). Assessment of microbiome changes after rumen transfaunation: implications on improving feed efficiency in beef cattle. Microbiome 6:62. doi: 10.1186/ s40168-018-0447-y

Conflict of Interest: The authors declare that the research was conducted in the absence of any commercial or financial relationships that could be construed as a potential conflict of interest.

Publisher's Note: All claims expressed in this article are solely those of the authors and do not necessarily represent those of their affiliated organizations, or those of the publisher, the editors and the reviewers. Any product that may be evaluated in this article, or claim that may be made by its manufacturer, is not guaranteed or endorsed by the publisher.

Copyright (c) 2022 Han, Xue, Cao, Chen, Qi, Ma, Tu, Diao, Zhang and Cui. This is an open-access article distributed under the terms of the Creative Commons Attribution License (CC BY). The use, distribution or reproduction in other forums is permitted, provided the original author(s) and the copyright owner(s) are credited and that the original publication in this journal is cited, in accordance with accepted academic practice. No use, distribution or reproduction is permitted which does not comply with these terms. 